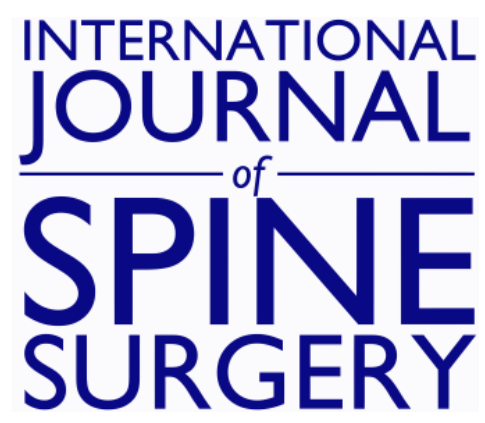

\title{
Is Degenerative Spondylolisthesis a Contraindication for Total Disc Replacement? Kineflex Lumbar Disc Replacement in 7 Patients With 24-Month Follow-up
}

Ulrich R. Hähnle, Karen Sliwa, Malan de Villiers, Ian R. Weinberg, Barry M.B.E. Sweet and Geoffrey P. Candy

Int J Spine Surg 2008, 2 (2) 92-100

doi: https://doi.org/10.1016/SASJ-2007-0125-NT

http://ijssurgery.com/content/2/2/92

This information is current as of April 25, 2023.

Email Alerts Receive free email-alerts when new articles cite this article. Sign up at: http://ijssurgery.com/alerts 


\title{
Is Degenerative Spondylolisthesis a Contraindication for Total Disc Replacement? Kineflex Lumbar Disc Replacement in 7 Patients With 24-Month Follow-up
}

\author{
Ulrich R. Hähnle, MD, FCS (Ortho), ${ }^{a, b}$ Karen Sliwa MD, PhD, ${ }^{a}$ Malan de Villiers, PhD, ${ }^{c}$ \\ Ian R. Weinberg, MD, FCS (Neuro), ${ }^{b}$ Barry M.B.E. Sweet, MD, PhD, ${ }^{a}$ and Geoffrey P. Candy, PhD ${ }^{a}$
}

\begin{abstract}
Background

Degenerative spondylolisthesis is associated with a significant segmental kyphosis at the level of the listhesis. We treated 7 disc spaces with Grade 2 listhesis and/or kyphosis of the slipped disc level with Kineflex disc replacement.
\end{abstract}

\section{Methods}

Out of a single-center prospective registry, involving 310 lumbar disc replacement patients, 7 patients underwent a single-level Kineflex disc replacement at the level of a degenerative spondylolisthesis with either segmental kyphosis or a Grade 2 slip.

Preoperative and follow-up radiological parameters studied were: pelvic incidence, pelvic tilt, sacral slope, lumbar lordosis L1-S1, degree of segmental listhesis, segmental lordosis, and range of motion (ROM). Clinical outcome measures were Visual Analog Scale pain score (VAS), Oswestry Disability Index (ODI), and patient satisfaction.

Results

Five replacements were performed at the L4-L5 level, and 2 were performed at a L3-4 level, above a pre-existing L4-S1 posterolateral fusion. Mean age was 50 (32-62) years. Average follow-up was $23.8 \pm 13.1$ months. Six of 7 patients considered their outcome as good or excellent. The mean VAS score decreased from $8.4 \pm 1.9$ to $2.7 \pm 2.2(P<.01)$. The ODI decreased from $45.2 \pm 9.9$ preoperatively to $19.7 \pm 12.8(P<.01)$.

There were increases in lumbar lordosis (from $47.40 \pm 10.6$ to $61.3 \mathrm{o} \pm 8.0(P<.03)$ ), in segmental lordosis $\left(\right.$ from $0.17^{\circ} \pm 7.0^{\circ}$ to $16.4^{\circ} \pm 2.0^{\circ}(P<.03)$ ), and in sacral slope (from $34.5^{\circ} \pm 4.8^{\circ}$ to $40.7^{\circ} \pm 4.5^{\circ}(P<.03)$ ). There were decreases in pelvic tilt (from $22.6^{\circ} \pm 6.3^{\circ}$ to $15.5^{\circ} \pm 5.9^{\circ}(P<.05)$ ), and degree of segmental listhesis (from $24.4 \% \pm 7.7$ to $3.7 \% \pm 3.4(P<.03)$ ). Pelvic incidence and ROM did not change.

\section{Conclusions}

Disc replacement resulted in significant improvement in clinical outcome and excellent sagittal balance and slip correction. However, the influence of improved sagittal spinal alignment on clinical outcomes needs to be investigated in larger studies including a control group.

\section{Clinical Relevance}

This study is the first focused on disc replacement in degenerative spondylolisthesis.

Key Words: Spondylolisthesis, total disc replacement, radiological outcome, clinical outcome. SAS Journal. Spring 2008;2:92-100. DOI: SASJ2007-0125-NT

\footnotetext{
${ }^{a}$ University of the Witwatersrand, Johannesburg, South Africa; ${ }^{b}$ Linkfield Park Clinic, Johannesburg, South Africa; ${ }^{c}$ University of Potchefstroom, South Africa

Address correspondence to Ulrich R. Hähnle, University of the Witwatersrand, PO-Box 52040, Saxonwold 2132, Johannesburg, South Africa (e-mail: hahnleu@mdh-africa.org)
}

Ulrich R. Hähnle, Ian R. Weinberg, and Malan de Villiers are co-developers of the Kineflex Disc Prosthesis and shareholders in Spinal Motion (Mountainview, California)

Institutional Review Board approval was obtained from the University of the Witwatersrand Ethics Committee. 


\section{INTRODUCTION}

Degenerative spondylolisthesis (DSPL) is a condition where degenerative changes in disc and facet joint complex lead to vertebral displacement, resulting in spinal stenosis, recess stenosis, and segmental kyphosis. ${ }^{1}$ Reports are mostly retrospective, and randomized studies have only compared surgical treatment consisting of posterolateral fusion with or without instrumentation and with posterior decompression alone. $^{2}$

The influence of sagittal alignment on the generation of lower back pain (LBP) and degeneration of the lower back is not well understood. Despite existing suspicion that preexisting differences in sagittal alignment may influence the occurrence of LBP and that outcome of fusion surgery may be dependent on restoration of lumbar lordosis during surgery, ${ }^{3}$ only recently a classification system to measure and classify sagittal alignment has been published. ${ }^{4}$ It has been applied to pathological conditions of the lumbar spine such as DSPL. ${ }^{5}$

Anterior lumbar interbody fusion (ALIF) surgery reliably corrects sagittal imbalance and listhetic slip in significant segmental kyphosis associated with DSPL. ${ }^{1,6,7}$ Anterior column support was recommended by Sengupta and Herkowitz for patients with Grade 2 spondylolisthesis or higher or when kyphosis was present. ${ }^{8}$

Dynamic posterior motion preservation in DSPL renders significant clinical improvement despite minimal sagittal alignment changes ${ }^{2,9-12}$ and despite increase in facet arthrosis. $^{11}$

Despite the potential positive effect on spinal alignment and degree of spondylolisthesis, significant DSPL is considered a contraindication for total disc replacement (TDR). Complications from inadvertently instrumented spondylolytic disc spaces have been presented, but objective confirmation of the outcome of TDR in DSPL is missing.

The Kineflex disc prosthesis (Spinal Motion; Mountainview, California) is a chrome-cobalt-molybdenum (BioDur CCM Plus; Carpenter Technology Corp., Wyomissing, Pennsylvania), unconstrained but recentering disc prosthesis with a variable center of rotation. The mechanism comprises 2 metal endplates articulating over a sliding core, which is positioned between the endplates. It allows $12^{\circ}$ of movement into flexion, extension, and left- and right-sided bending. The inferior endplate has a retaining ring that limits the excursion in the inferior articulation to $2 \mathrm{~mm}$ in all directions and prevents dislodgement of the sliding core. The mechanism therefore only allows $4 \mathrm{~mm}$ of translation before distraction of the disc space; a recentering force is produced that counteracts the translation. The disc is inserted as a single unit with a freely mobile mechanism during the final insertion process to facilitate placement posteriorly within the disc space. The objective in the development of this prosthesis was to facilitate reliable midline and posterior placement of the implant within the disc space in severely degenerative disc spaces, through a minimally invasive approach. ${ }^{13}$

The insertion technique of this disc prosthesis is unique. After the initial engagement into the disc space of the fully assembled 3-component prosthesis, the insertion tools allow independent advancement of the superior and inferior prosthetic endplates. During this process the advancing endplate pivots over the sliding core, taking pressure off the leading prosthetic endplate/bone interphase. ${ }^{13}$ We therefore postulated that, through independent advancement of the inferior endplate, this particular disc prosthesis should be able to assist spondylolisthesis reduction during the insertion process.

We are reporting on the operative reduction technique in DSPL and on the outcome of 7 patients with either a Grade 2 spondylolisthesis and/or kyphosis of the slipped disc level and who were treated with Kineflex disc replacement.

\section{MATERIAL AND METHODS}

Out of a single-center prospective registry involving 310 lumbar disc replacement patients, 7 patients were retrieved from our databank of patients who had undergone a single-level Kineflex disc replacement at the level of a degenerative spondylolisthesis with either segmental kyphosis or a Grade 2 slip.

\section{Operative Technique}

The operations were performed through a left-sided retroperitoneal approach, followed by the creation of a wide exposure of the disc space. After a midline anuloplasty, a complete nucleotomy was performed, and the inner, desiccated layers of the annulus were removed. The disc space was mobilized, and the bony endplates were prepared. The correct-sized prosthesis was selected. As hypermobility was an anticipated complication, the disc height selected was one size larger than we would have chosen in a standard disc replacement. After initial engagement of the prosthesis, the mechanism of the prosthesis was released, and the endplates were advanced until almost flush with the posterior wall of the inferior vertebral endplate of the cephalad vertebra. Thereafter, the inferior prosthetic endplate was further advanced until almost full spondylolisthesis reduction was achieved. Additional screw fixation of the inferior endplate was performed, whenever further primary fixation was thought to be necessary, in order to absorb excessive forces through the inferior prosthetic endplate/bone interphase (Figure 1).

\section{Radiographic Evaluation}

All patients had a preoperative magnetic resonance image (MRI) or lumbar myelography followed by computer tomography (CT), or both.

Preoperatively and postoperatively at 3 months, 6 months, and yearly, anteroposterior (AP), lateral standing radiographs that included the bottom endplate of the T12 vertebra and the 
Figure 1. Insertion Technique
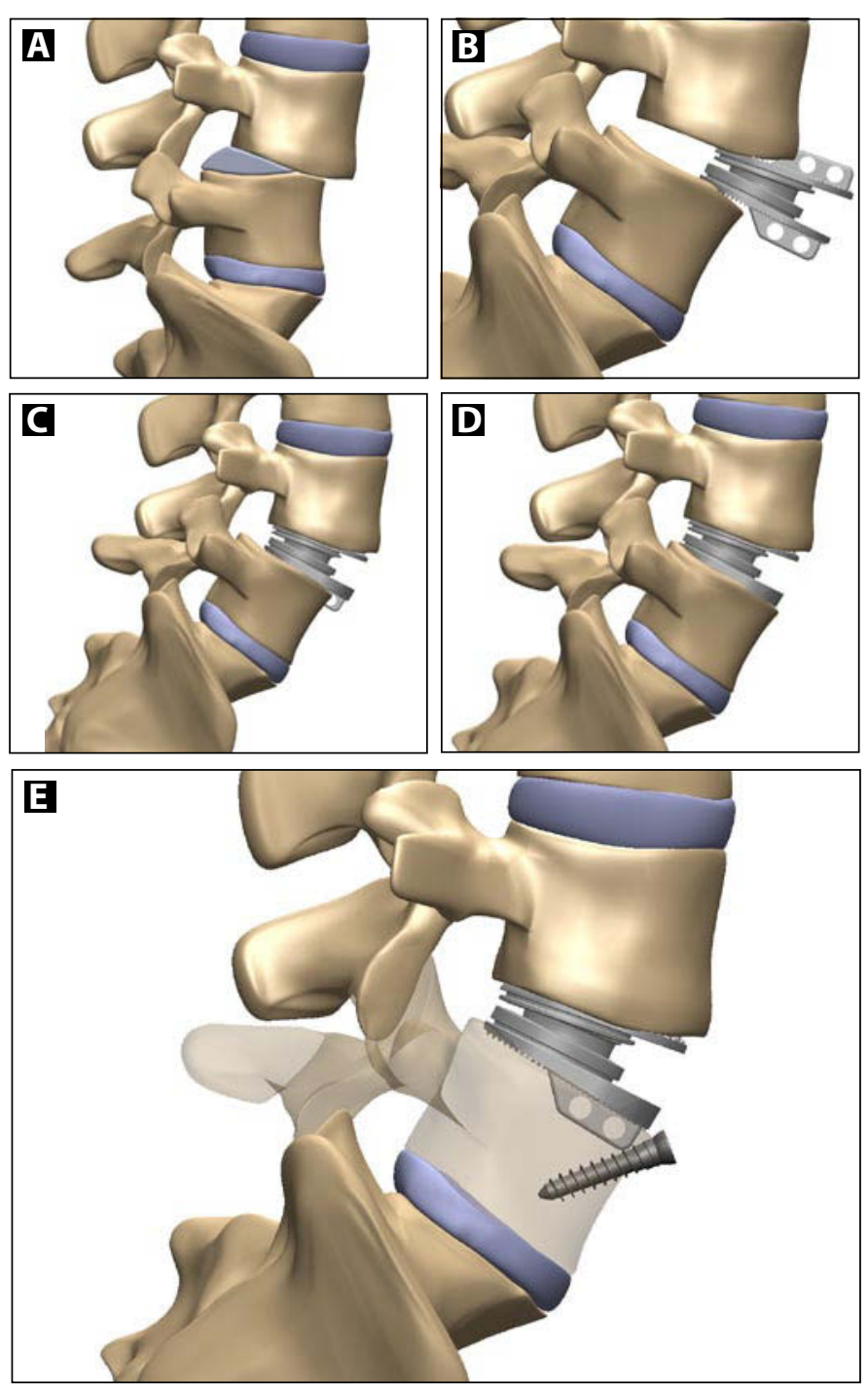

Position A: Spondylolisthetic disc space before operation. Position B: Initial engagement of the prosthesis into the disc space after full nucleotomy, disc space mobilization, and cutting of the insertion grooves. The further insertion is performed with a fully freed prosthetic mechanism. Position C: Advancement of the prosthesis into the disc space, until almost flush with the posterior wall of the cephalad vertebral body. The prosthetic endplates are individually advanced, alternating between top and bottom prosthetic endplate. Position D: Further advancement of the bottom prosthetic endplate, until almost flush with the posterior wall of the caudad; full reduction of the spondylolisthesis. During this advancement the inferior endplate pivots around the articulating mechanism of the prosthesis, reducing the pressure between the leading edge of the inferior prosthetic endplate and the bony endplate. Position E: Securing of the final reduced position by placement of a screw into the into the insertion groove of the inferior vertebra. This counteracts the displacing forces (For more information on insertion technique, see Hähnle et al.13)

top half of both femoral heads were performed. In addition, a lateral, whole-spine, standing radiograph was included. The patients were asked to stand straight with arms crossed over the chest and knees fully extended. Lateral flexion/extension radiographs were also performed at these follow-ups. The spinal balance evaluation was based on the studies of Duval Beaupere et al. ${ }^{14-16}$ and Lazennec et al. ${ }^{17}$ (Figure 2). We looked at pelvic incidence (PI), sacral slope (SS), pelvic tilt (PT),
Figure 2. Lumbar Disc Replacement in DSPL Measurements

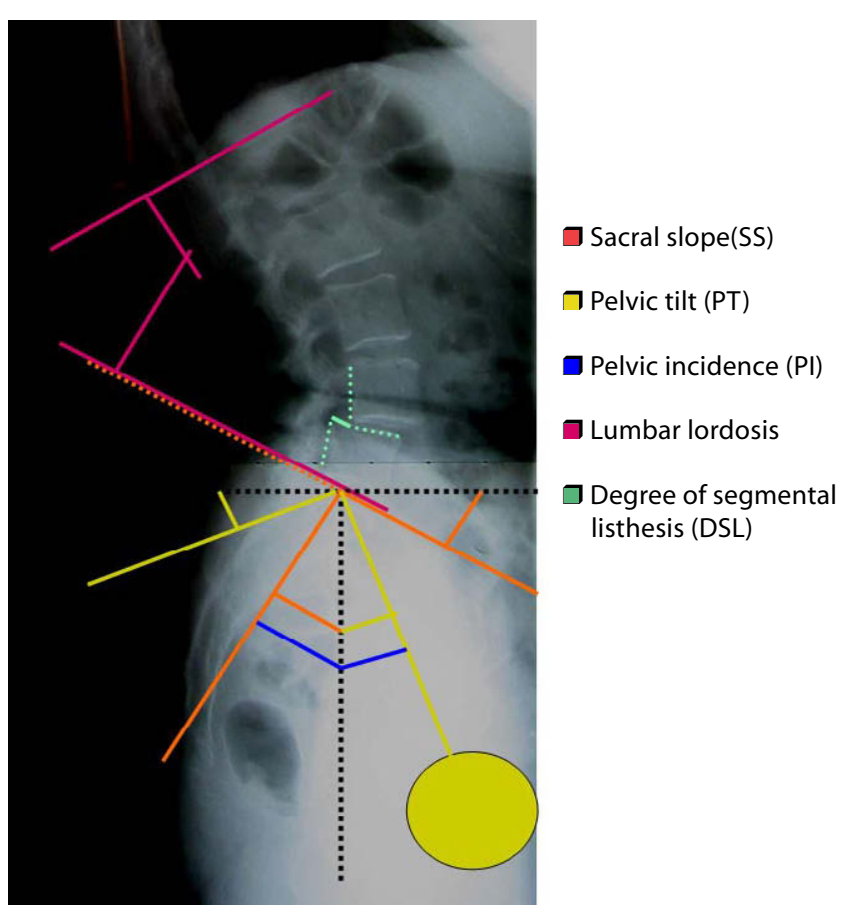

Lumbar disc in DSPL-measured parameters. Pelvic incidence (PI), sacral slope (SS); pelvic tilt (PT), lumbar lordosis (cephalad endplate L1-cephalad endplate S1) (LL)

lumbar lordosis (cephalad endplate L1-cephalad endplate S1) (LL), and segmental lordosis (SL) within the instrumented disc space. If the femoral heads were not exactly superimposed on each other, the middle of the line connecting the centers of the femoral heads was used to determine the PI and PT. We further studied the degree of segmental listhesis (DSL) on a percentage basis. The range of motion (ROM) at the level of DSPL was measured on flexion/extension radiographs by 2 of the investigators (U.H. and I.W.), preoperatively by the Cobb method and postoperatively by the fin method. ${ }^{18}$

\section{Clinical Evaluation}

Clinical outcome was measured using the visual analogue pain score (VAS), Oswestry Disability Index (ODI), ${ }^{19}$ patient satisfaction, and "return to work" data. Complications were described.

\section{Statistical Analysis}

Data were reported as mean \pm standard deviation with SAS V9.1 (SAS Institute Inc., Cary, North Carolina) for statistical analysis and comparisons. Changes in measured variables preand postoperatively were compared using a nonparametric Wilcoxon matched pairs test with a $P$ value $<.05$ regarded as significant.

\section{RESULTS}

Seven patients were retrieved from our databank who had undergone a single-level Kineflex disc replacement at the level of a spondylolisthesis with either segmental kyphosis or 
a Grade 2 slip or both and had undergone the index procedure at least 1 year before. They were called in for clinical and radiological follow-up; these patients form the base of this study.

\section{Figures $3 A$ and $3 B$.}

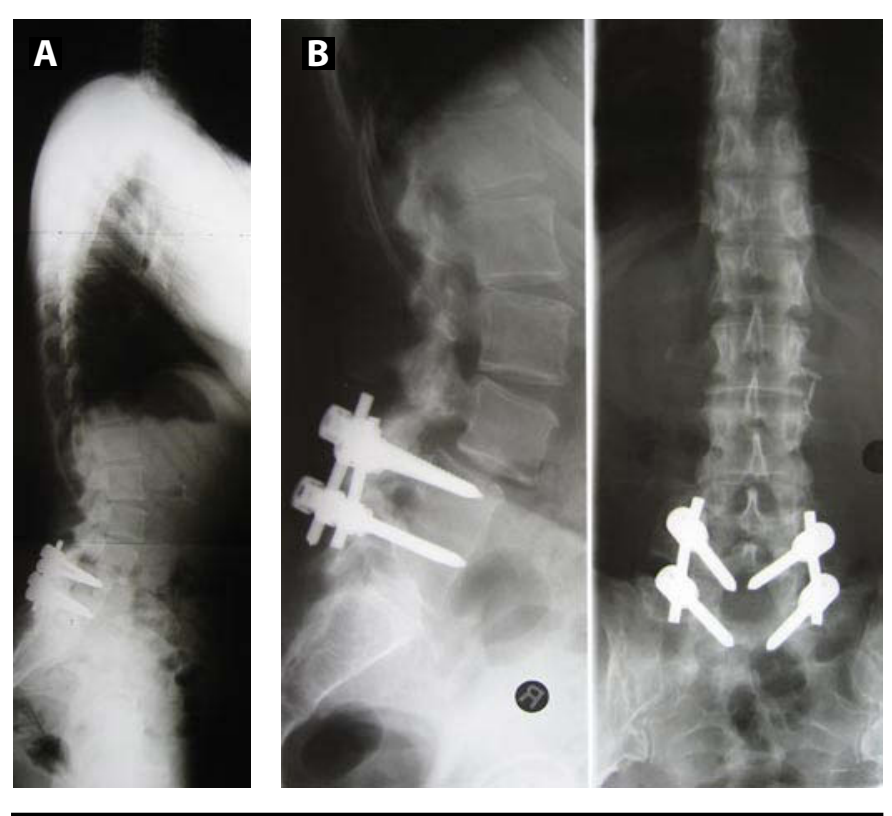

43-year-old active lady with severe LBP and SS symptoms. Posterolateral fusion L4-S1 4 years before.

\section{Figures 3C and 3D.}
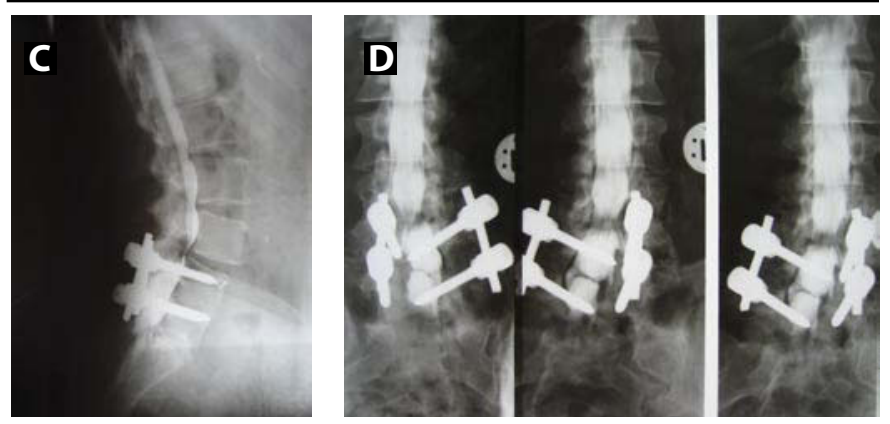

Myelogram confirms severe SS

Five disc replacements had been performed at the L4-L5 lumbar level. In one operation a posterior motion preservation device, inserted 2 years prior to the index operation, had first been removed through a posterior approach before the prosthetic disc insertion during the same anesthetics. Two disc replacements had been performed at a L3-L4 level, above a pre-existing L4-S1 posterolateral fusion (Figure 3 ). The average age at the time of the index procedure was $50(32-62)$ years. Five of the patients were female. All patients had originally presented with symptoms of mechanical LBP and leg pain. Five patients had complained with symptoms of spinal stenosis.
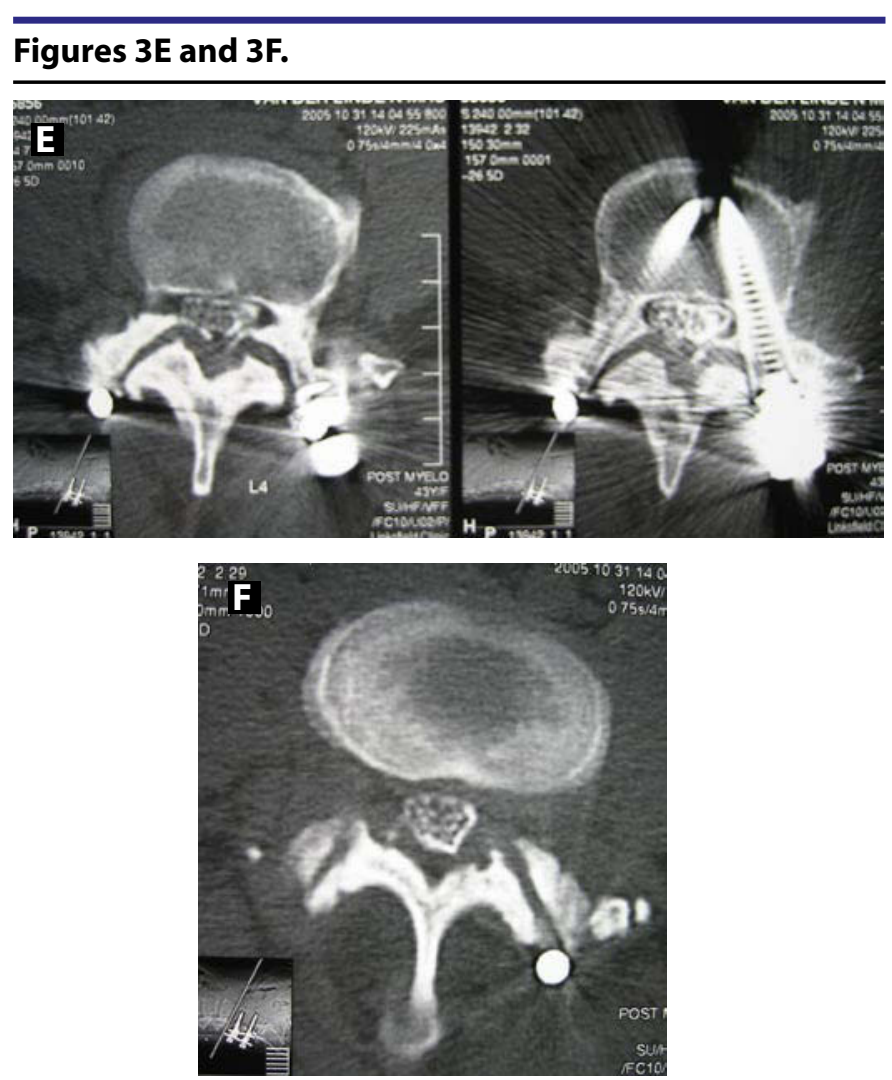

CT Myelogram shows severe facet arthrosis.

One of the 7 patients had a previous posterior dynamic system inserted by another surgeon 2 years prior to our operation. This person is not a DSPL patient, but the previous surgery resulted in a radiologically similar picture. The operation had left her with significant posterior distraction, segmental kyphosis, and instability. She refused fusion surgery. We therefore performed posterior implant removal followed by anterior TDR surgery.

The postoperative hospital stay averaged 3.3 days (2-8 days) with all patients returning to work after 27 days (3-42 days). The average follow-up was $23.8 \pm 13.1$ months. One of the patients considered the outcome as fair, 2 as good, and 4 as excellent. Six patients "would undergo the operation again," and one "doesn't know." The VAS score decreased from $8.4 \pm 1.9$ to $2.7 \pm 2.2$. The ODI decreased from $45.2 \pm 9.9$ preoperatively to $19.7 \pm 12.8$.

One patient was reoperated at 1 week postoperatively for partial extrusion of the inferior prosthetic endplate over the malpositioned buttress screw (Figure 4). One patient developed a left iliac deep vein thrombosis (DVT).

The lumbar lordosis (LL), segmental lordosis (SL), and sacral slope (SS) increased. LL increased from $47.4^{\circ} \pm 10.6^{\circ}$ to $61.3^{\circ}$ $\pm-8.0^{\circ}(P<.03)$; SL from $0.17^{\circ} \pm 7.0^{\circ}$ to $16.4^{\circ} \pm 2.0^{\circ}(P<$ $.03)$; and SS from $34.5^{\circ} \pm 4.8^{\circ}$ to $40.7^{\circ} \pm 4.5^{\circ}(P<.03)$. The pelvic tilt (PT) and the degree of segmental listhesis (DSL) 


\section{Figures $3 \mathrm{G}, 3 \mathrm{H}, \mathbf{3 I}, \mathbf{3} \mathrm{J}$, and $3 \mathrm{~K}$.}
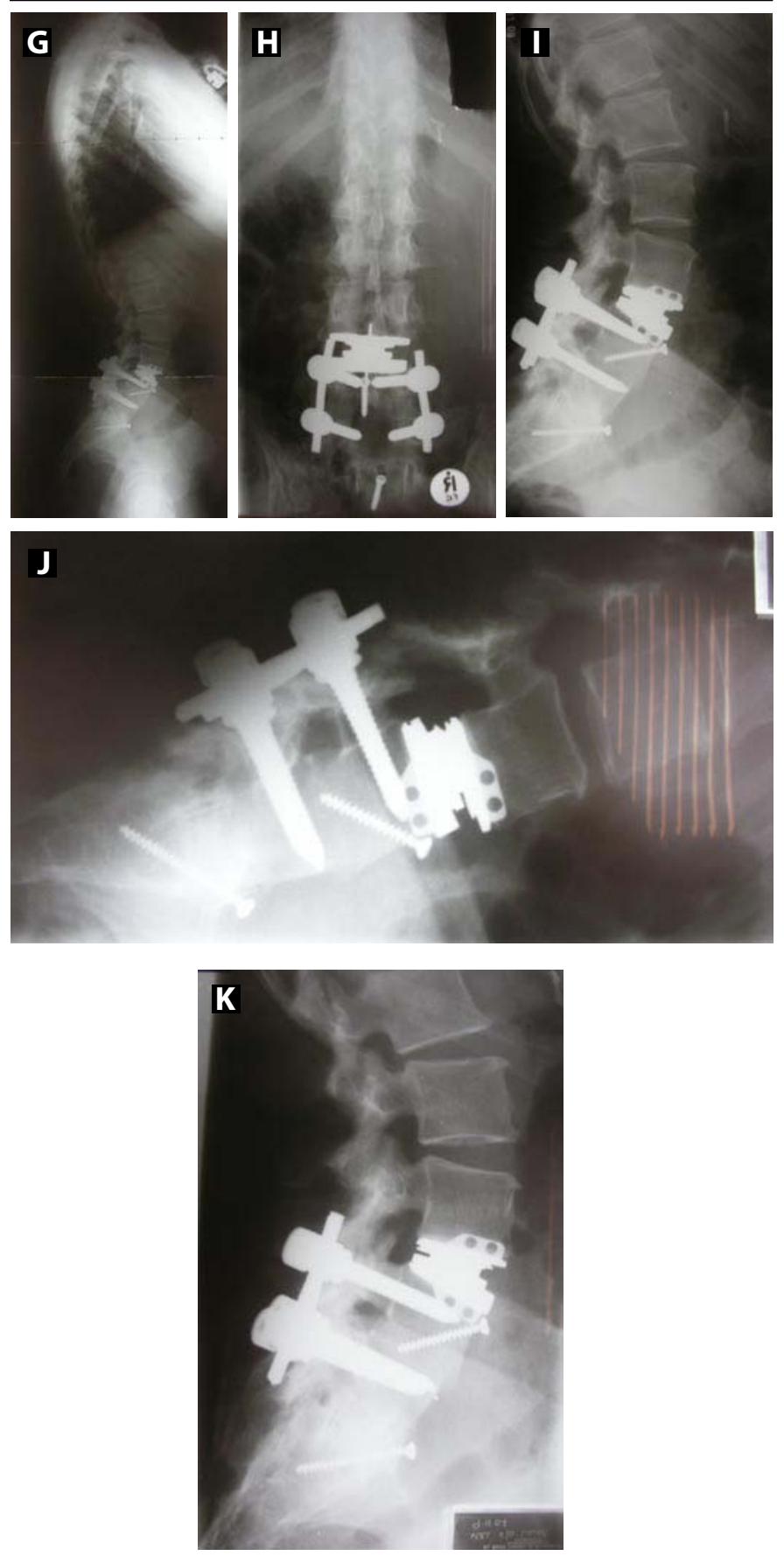

2-year follow-up: good clinical $(\mathrm{ODI}=22, \mathrm{VAS}=2)$ and radiological outcome.

decreased. PT decreased from $22.6^{\circ} \pm 6.3^{\circ}$ to $15.5^{\circ} \pm 5.9^{\circ}$ $(P<.05)$; DSL from $24.4 \% \pm 7.7$ to $3.7 \% \pm 3.4(P<.03)$. The pelvic incidence (PI) did not change (preoperative PI was 56.25 while the latest follow-up was 56.5). The change in ROM was insignificant $\left(11.9^{\circ} \pm 5.0^{\circ}\right.$ preoperatively to $11.6^{\circ}$ $\pm 3.6^{\circ}$ at the latest follow-up). Due to the small cohort no correlation could be drawn between clinical outcome and changes in radiological parameters.

\section{DISCUSSION}

The etiology of degenerative spondylolisthesis (DSPL) is poorly understood. Sagittal orientation of the facet joint has been implicated as a predisposing factor ${ }^{20}$ but might be instead a consequence of the remodelling taking place during development of facet arthritis. ${ }^{21}$ Controversy exists as to whether the pathology starts primarily in the facet joints ${ }^{22}$ or within the intervertebral disc. ${ }^{1}$ Whatever the etiology, the result is segmental spondylolisthesis, segmental kyphosis, spinal and recess stenosis, and facet joint arthritis.

Our cohort of patients consisted of 3 different entities: degenerative spondylolisthesis proper, adjacent segment disease (ASD) with resulting (degenerative) spondylolisthesis at the adjacent segment, and 1 iatrogenic spondylolisthesis caused by an over-distracted posterior device. The resulting clinical and radiological picture is very similar. Clinical patients complained about mechanical LBP and leg pain, inability to stand or walk longer distances. Radiologically, the deformity resulted in low lumbar flat back deformities with compensatory retrolisthesis of the higher lumbar motion segments and flexion of the pelvis on lateral standing radiographs. In each of the 3 entities the resulting surgical treatment aim is similar: decompression of neural structures, stabilization of the motion segment, and restoration of the spinal balance. As we are primarily describing a surgical technique aimed to treat these different components, we considered it justifiable to sum these patients together.

Most surgical outcome studies on DSPL were retrospective and not controlled. Randomised studies have only compared surgical treatment consisting of posterolateral fusion with or without instrumentation and with posterior decompression alone. ${ }^{2}$ Long-term outcome of surgical treatment seems more favorable with fusion than without fusion. ${ }^{23,24}$

Anterior lumbar interbody fusion (ALIF) surgery reliably corrects sagittal imbalance and listhetic slip in significant segmental kyphosis associated with DSPL. ${ }^{1,6,7}$ Anterior column support was recommended by Sengupta and Herkowitz for patients with Grade 2 slips or higher or when frank kyphosis is present. ${ }^{8}$

More recently Choi and Sung reported on a large patient group, 14 patients with DSPL, using single-level standalone rectangular cages. At 27 months follow-up there was subsidence of over $2 \mathrm{~mm}$ in $77 \%$ of patients and a $13 \%$ nonunion rate, but neither correlated with recurrence of symptoms. ${ }^{25}$ In ALIF there is a nonunion rate which might be asymptomatic, ${ }^{25}$ but in case of a later established symptomatic anterior nonunion, supplementation with an instrumented posterior fusion does not always relieve the clinical symptoms. ALIF, as a standalone procedure, has a risk of anterior dislodgement in spondylolisthesis. Furthermore, the primary contact area of fusion cages is far less than the endplate surface area of the disc prosthesis. 
Figures 4A, 4B, and $4 C$.

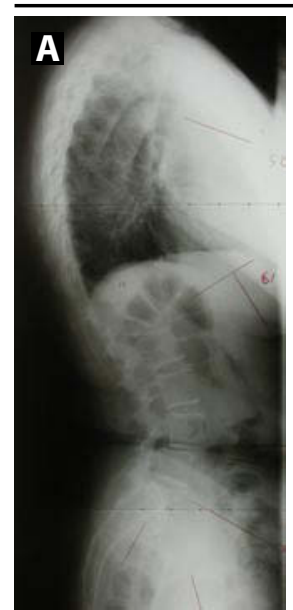

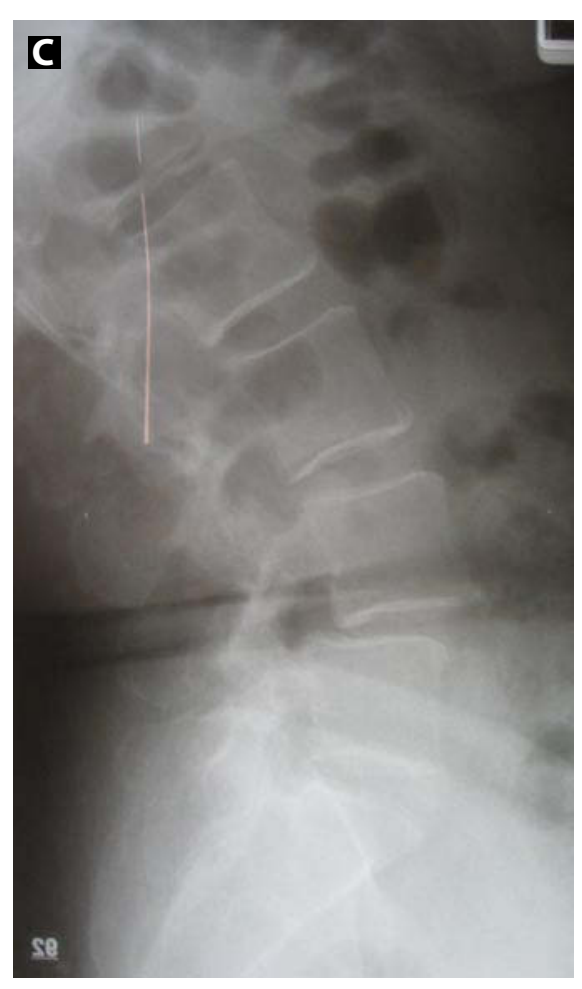

62-year-old patient with severe mechanical LBP and right-side leg pain for 2 years.

Brantigan et al. ${ }^{26}$ reported 10 -year results on a subgroup of an original US Food and Drug Administration trial, using carbon fiber posterior lumbar interbody fusion (PLIF) cages in conjunction with a posterior screw and plating system $\left(360^{\circ}\right.$ fusion) with excellent clinical and radiological outcomes. McAfee et al. ${ }^{27}$ reported on 120 patients with spondylolisthesis, half of them with DSPL, who were treated with a 360 -degree fusion through a single posterior approach with a $98 \%$ fusion rate, good radiological outcome, and few complications.

In a prospective study, Konno and $\mathrm{Kikuchi}^{9}$ compared surgical decompression, together with posterior dynamic Graf ligament stabilization, to decompression surgery alone. They found better clinical outcome in the Graf ligament group. Significant clinical improvement was confirmed in other studies using dynamic stabilization, ${ }^{2,10-12}$ despite an increase in facet

\section{Figures $4 \mathrm{D}$ and $4 \mathrm{E}$.}
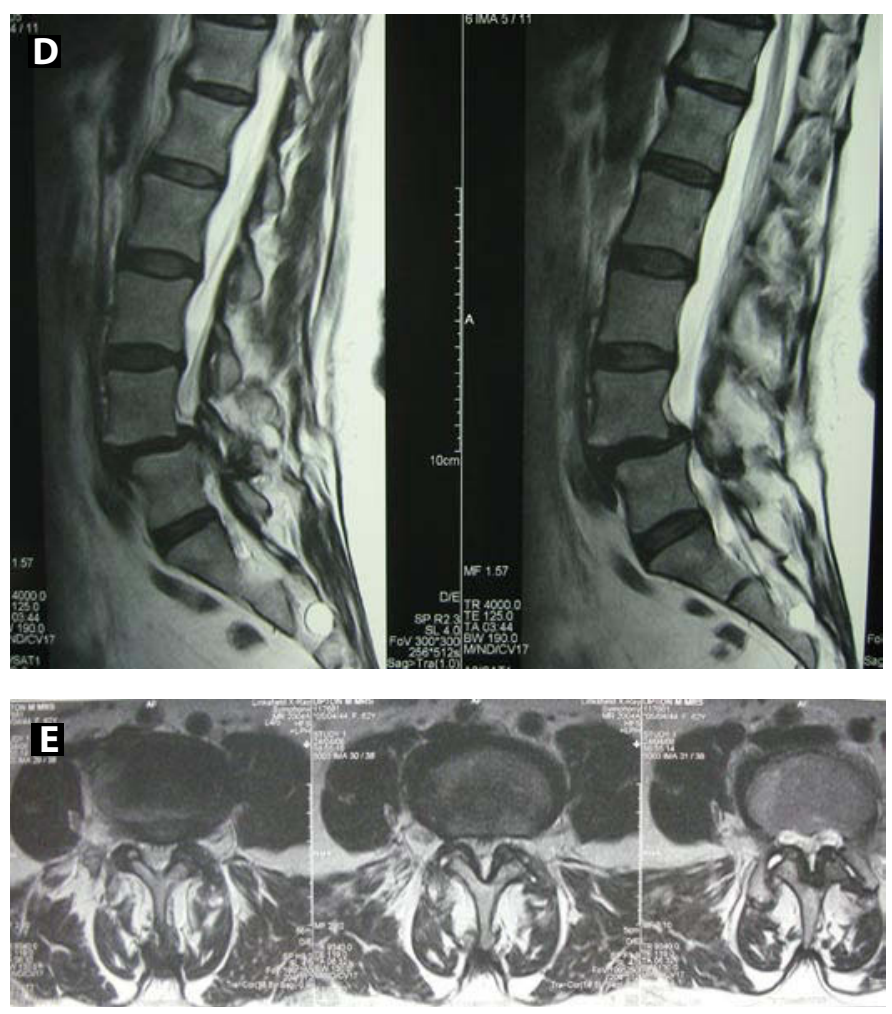

MRI scanning shows partial reduction of listhesis in lying position, significant L4-L5 facet degeneration and DDD.

\section{Figure 4 F.}

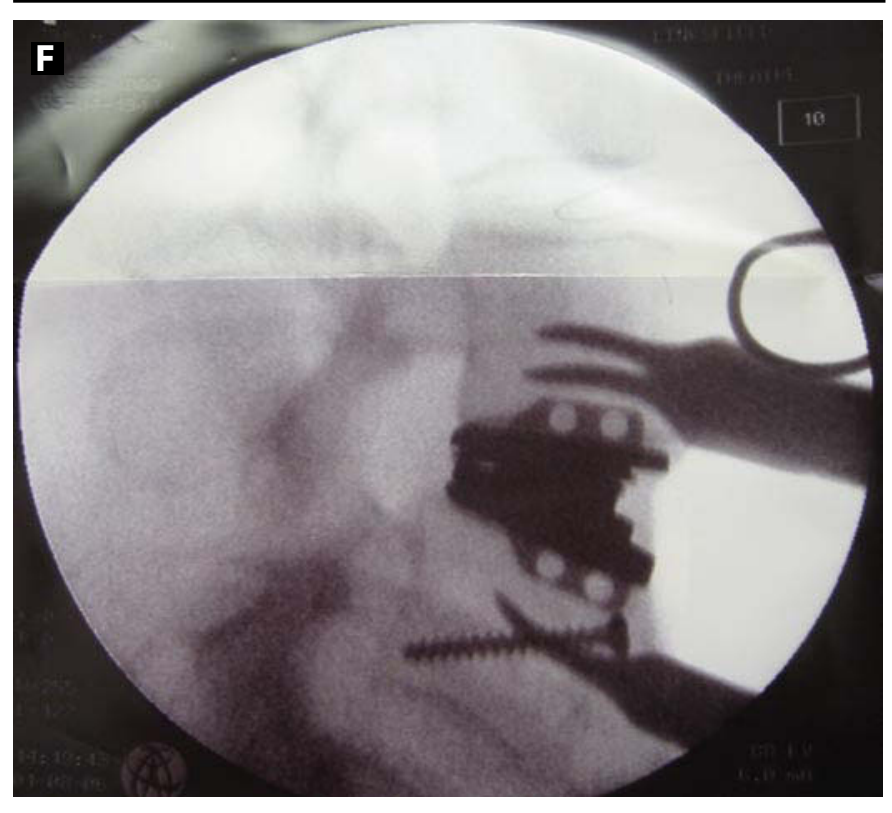

Intraoperative radiograph shows good alignment and listhesis reduction.

arthrosis. ${ }^{11}$ Posterior tension-band-like devices, in the absence of anterior support, should increase the load on the facet joints, and they are unable to significantly improve sagittal 


\section{Figures 4G and $4 \mathrm{H}$.}

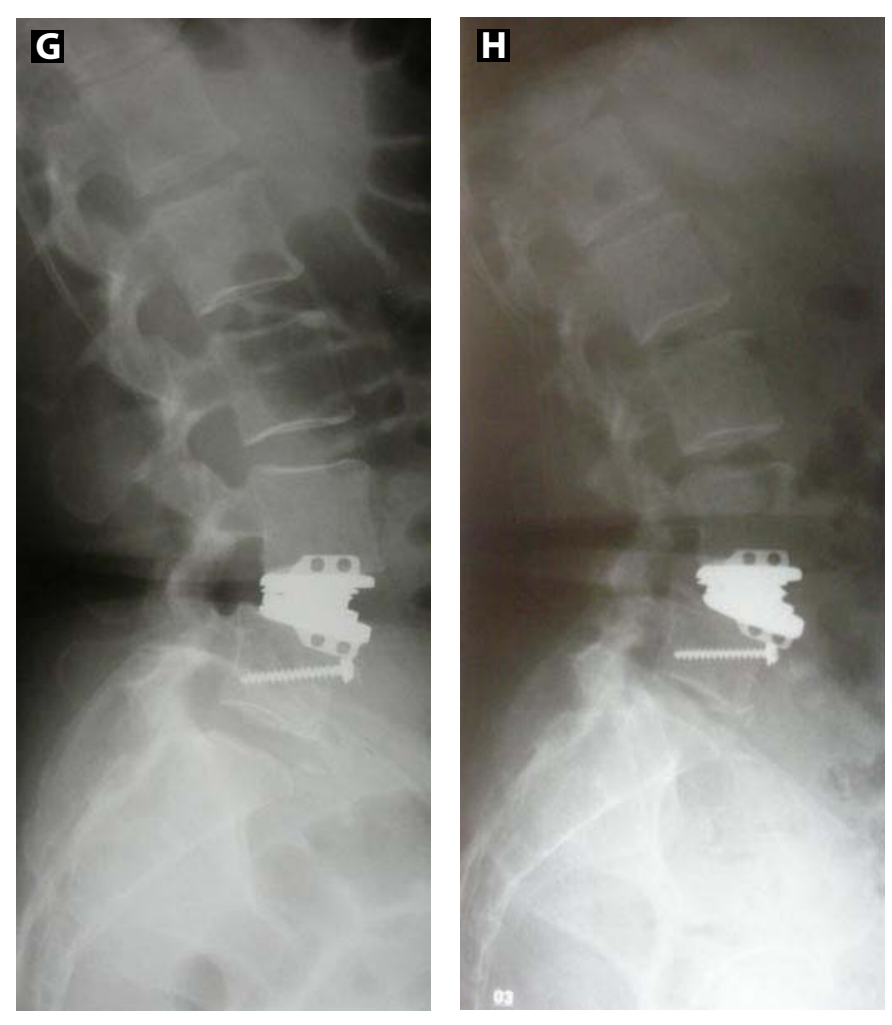

(G) Partial extrusion of inferior prosthetic endplate at 2 days. (H) Further extrusion of inferior prosthetic endplate at 6 days.

alignment. Taking this into consideration, the improvement in clinical outcome in these patients is remarkable. Recently McAfee et al. reported clinical improvement with a motionpreserving facet replacement system (TOPS). ${ }^{28}$

Despite the success with posterior motion-preserving techniques in DSPL, even in the absence of spondylolisthesis and kyphosis reduction, ${ }^{2,10-12}$ DSPL over $3 \mathrm{~mm}$ is considered a contraindication for TDR. ${ }^{27}$ Nevertheless, objective confirmation of the outcome of TDR in DSPL is missing. Considerable reduction of the spondylolisthesis should be desirable in order to restore the normal 3-joint anatomy (disc and 2 facet joints) of the motion segment. This could not be achieved with posterior motion-preserving procedures. ${ }^{2,10-12}$

The potential advantages of disc replacement with an unconstrained disc and non-rigidly held prosthetic endplates during insertion are, at least in theory, the following (see also Figure 1 and Hähnle et al. ${ }^{13}$ ):

1) During the insertion process the prosthetic endplates can follow the shape of the bony endplates as they are able to pivot around the mechanism. The leading edge of a rectangular cage, or rigidly held disc prosthesis, is more likely to be impacted into the bony endplate, possibly resulting in endplate fracture and subsidence.
2) Once the Kineflex disc prosthesis is almost lying flush with the posterior vertebral body wall of the cephalad vertebra, the inferior endplate can be further advanced to facilitate reduction of the spondylolisthesis. As during this process part of the impact of the further advancement is absorbed in the prosthetic mechanism, the cephalad prosthetic endplate stays behind, avoiding protrusion of the superior prosthetic endplate into the spinal canal. With a cage or an unconstrained disc prosthesis, this manuever would easily result in impaction of the implant into the posterior part of the vertebral endplate of the cephalad vertebra (endplate fracture) or in protrusion of the implant into the spinal canal.

3) During mobilization of the patient the forces that could lead to expulsion of the inferior prosthetic endplate are at least partially dampened within the prosthetic mechanism, which allows translation but at the same time is recentering. Furthermore, flexion/extension motion through the operated segment does not lead to cyclical loss of contact (rocking) of the implant/bone surfaces and possible nonunion or nonintegration, as the contact is maintained by the adaptive movement of the prosthetic endplate.

\section{Figures 4I, 4J, 4K, and 4L.}
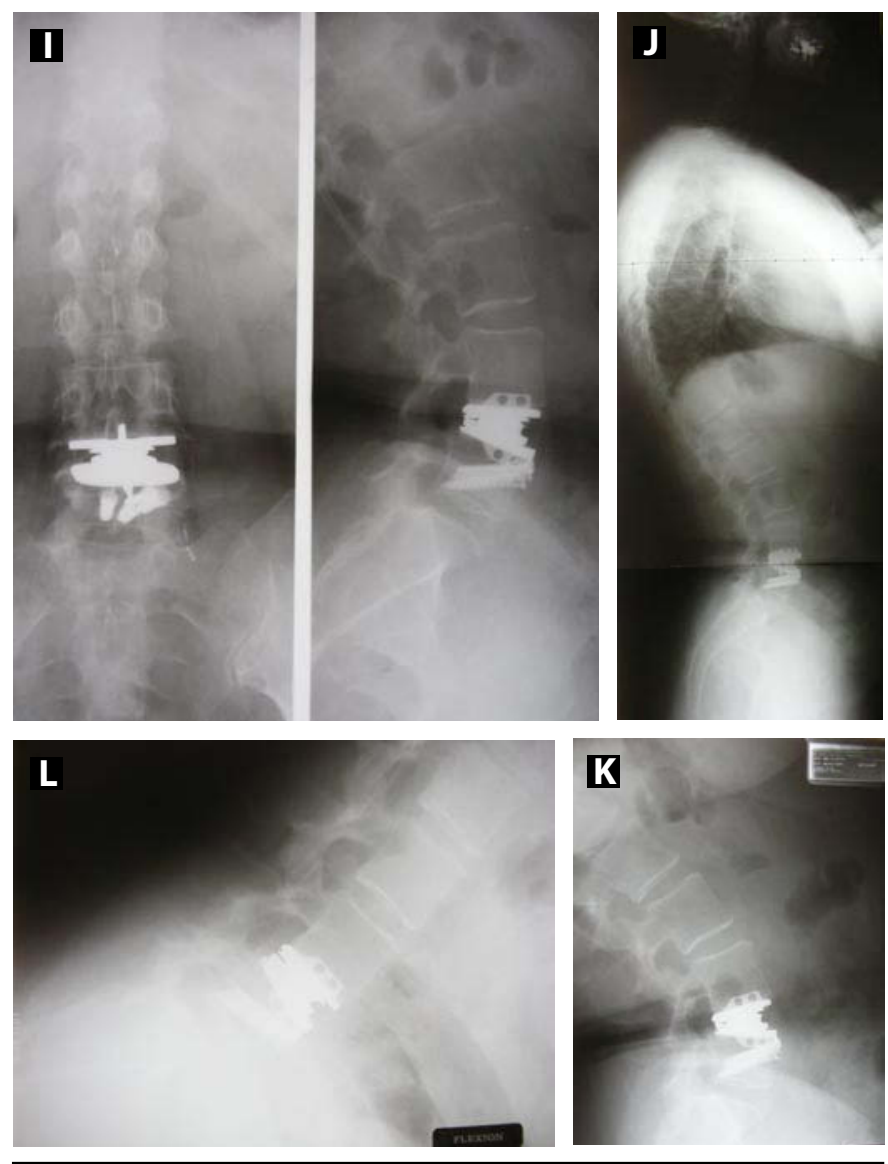

2 years after reoperation to reposition inferior endplate: excellent clinical $(\mathrm{ODI}=0 ; \mathrm{VAS}=0)$ and radiological outcomes. 
With the Kineflex insertion technique used, we achieved excellent sagittal balance and slip correction in all patients. The continuous advancement of the inferior prosthetic endplate, after seating the prosthesis close to the posterior edge of the apical vertebral endplate, allowed additional reduction of the spondylolisthesis. The postoperatively high translational forces on the inferior prosthetic endplate could be counteracted with additional screw fixation used as a buttress. No postoperative hypermobility developed at the replaced levels. One reoperation was performed early after the index procedure with excellent clinical and radiological final outcome (Figure 4).

The most common level in DSPL is the L4-5 motion segment. At this level dislodgement of the implant would be of particular concern because of the proximity of important vascular structures. We would generally try to avoid anterior revision surgery at this level after 17-18 days and would rather wait past 3 months when the postoperative inflammation has completely settled. We therefore performed our anterior revision surgery for partial endplate extrusion early, after the first follow-up at 8 days postoperatively, despite good clinical relief of symptoms at that early stage (Figure 4).

The indications for TDR have hardly been modified since the onset of modern type TDR over 20 years ago. ${ }^{29}$ Only recently Siepe et al. ${ }^{30}$ published clinical outcome of TDR dependent on indications. There is a need for arthroplasty surgeons to publish results of off-label TDR procedures, in order to establish their validity for different indications and in order to learn from the experience of other surgeons.

\section{CONCLUSION}

This is a pilot study with significant limitations: Only 7 patients were involved with no control group, and the follow-up is short. Early results are promising, but because of the limitations, one has to be careful about the clinical outcome results. With the insertion technique of the tested device we achieved excellent sagittal balance and slip correction in all patients. No postoperative hypermobility developed at the replaced levels. The influence of improved sagittal spinal alignment on clinical outcome needs to be investigated in larger studies.

This manuscript was submitted December 16, 2007, and accepted for publication April 21, 2008.

Acknowledgement: The authors thank Stephanie Hanekom and Aldorin Gehring for their enthusiastic support with patient management and follow-up.

\section{REFERENCES}

1. Inoue S, Watanabe T, Goto S, et al. Degenerative spondylolisthesis: Pathophysiology and results of anterior interbody fusion. Clin Orthop Relat Res. 1988;227:90-98.

2. Mochida J, Suzuki K, Chiba M. How to stabilize a single level lesion of degenerative lumbar spondylolisthesis. Clin Orthop Relat Res. 1999;368:126-134.
3. Izumi Y, Kumano K. Analysis of sagittal lumbar alignment before and after posterior instrumentation: Risk factor for the adjacent unfused segment. Eur J Orthop Surg. Traum. 2001;1:9-13.

4. Roussouly P, Gollogly S, Berthonnaud E, et al. Classification of normal variation in the sagittal alignment of the human lumbar spine and pelvis in the standing position. Spine. 2005, 30;3:346-353.

5. Barrey C, Jund J, Noseda O, et al. Sagittal balance of the pelvis-spine complex and lumbar degenerative diseases. A comparative study about 85 cases. Eur Spine J. 2007;16:1459-1467.

6. Takahashi K, Kitahara H, Ando M, et al. Long-term results of anterior interbody fusion for treatment of degenerative spondylolisthesis. Spine. 1990;15:1211-1215.

7. Santomi K, Hirabayashi K Toyama Y, et al. A clinical study of degenerative spondylolisthesis: radiographic analysis and choice of treatment. Spine. 1992;17:1329-1336.

8. Sengupta DK, Herkowitz HN. Degenerative Spondylolisthesis: Review of current trends and controversies. Spine. 2005;30:S71-S81.

9. Konno S, Kikuchi S. Prospective study of surgical treatment of degenerative spondylolisthesis: comparison between decompression alone and decompression with Graf system stabilisation. Spine. 2000;25:1533-1537.

10. Matsudaira K, Yamazaki T, Seichi A. Spinal stenosis in grade I degenerative lumbar spondylolisthesis: a comparative study of outcomes following laminoplasty and laminectomy with instrumented spinal fusion. J Orthop Sci. 2005;10:270-276. DOI 10.1007/s00776-005-0887-7.

11. Kanayama M, Hashimoti T, Shigenobu K, et al. Non-fusion surgery for degenerative spondylolisthesis using artheficial ligament stabilisation: surgical indication and clinical results. Spine. 2005;30:588-592.

12. Shim CS, Lee S-H, Park S-H, et al. Soft stabilisation with an artificial intervertebral ligament in grade I degenerative spondylolisthesis: comparison with instrumented posterior lumbar interbody fusion. $S A S$ Journal. Summer 2007;1:118-124. DOI: SASJ-2006-0006-RR.

13. Hähnle UR, Weinberg IR, Sliwa K, et al.: Kineflex (Centurion) lumbar disc prosthesis: Insertion technique and 2-year clinical results in 100 patients. SAS Journal. Winter 2007;1:28-35. DOI: SASJ-2006-0005-RR.

14. Legaye J, Duval-Beaupere, Hecquet $\mathrm{J}$, et al. Pelvic incidence: a fundamental pelvic parameter for three-dimensional regulation of sagital curves. Eur Spine J. 1998;7:99-103.

15. Duval-Beaupere G, Schmidt C, Cosson P. A barycentremetric study of the sagital shape of the pelvis: the conditions required for an economic standing position. Ann Biomed Eng. 1992;20:451-462.

16. Boulay C, Tardieu C, Hecquet J, et al. Sagital alignment of the spine and pelvis regulated by pelvic incidence: standard values and prediction of lordosis. Eur Spine J. 2006;15:415-422.

17. Lazennec JY, Ramare S, Arafati N, et al. Sagital alignement in lumbosacral fusion: relation between radiological parameters and pain. Eur Spine J. 2000;9:47-55.

18. Cakir B, Richter M, Kafer W, et al. Reliability of motion meassurements after total disc replacement the spike and the fin method. Eur Spine J. 2006;15:165-173

19. Fairbank JC, Pynsent PB. The Oswestry Disability Index. Spine. 2000;25:2940-2952.

20. Rosenberg NJ. Degenerative spondylolisthesis: predisposing factors. $J$ Bone Joint Surg [Am]. 1975;57:467-474.

21. Love TW, Fagan AB, Frazer RD. Degenerative spondylolisthesis: developmental or aquired? J Bone Joint Surg [Br]. 1999;81:640-644.

22. Rothman SL, Glenn WV Jr, Kerber CW. Multiplanar CT in the evaluation of degenerative spondylolisthesis: a review of 150 cases. Comput Radiol. $1985 ; 9: 223-232$ 
23. Kornblum MB, Fischgrund JS, Herkowitz NH, et al. Degenerative lumbar spondylolisthesis with spinal stenosis: a prospective long-term study comparing fusion and pseudarthrosis. Spine. 2004;29:726-733.

24. Martin CR, Gruszczynski AT, Braunfurth HA, et al. The surgical management of degenerative lumbar spondylolisthesis. A systhematic review. Spine. 2007;32:1791-1798.

25. Choi JY, Sung KH. Subsidence after anterior lumbar interbody fusion using paired stand-alone rectangular cages. Eur Spine J. 2006;15:16-22.

26. Brantigan JW, Neidre A, Toohey JS. The Lumbar I /F Cage for posterior lumbar interbody fusion with the Variable Screw Placement System: $10 \mathrm{y}$ results of an Food and Drug Administration clinical trial. Spine J. 2004;4;681-688.

27. McAfee PC, DeVine JG, Chaput CD, et al. The indication for interbody fusion cages in the treatment of spondylolisthesis. Spine. 2005;30:S60S65.

28. McAfee PC, Khoo LT, Pimienta L, et al. Treatment of lumbar spinal stenosis with a total posterior arthroplasty prostesis: implant description, surgical technique, and a prospective report on 29 patients. Neurosurg Focus. 2007;22:E13.

29. Buettner-Janz K, Schellnack K, Zippel H. Eine alternative Behandlungsstrategie beim lumbalen Bandscheibenschaden mit der Bandscheiben-endoprothese Modulartyp SB Charite. Z Orthop. 1987;125:1-6.

30. Siepe CJ, MayerHM, Wiechert K, et al. Clinical results of lumbar disc replacement with Pro-Disc II: three-year results for different indications. Spine. 2006;31:1923-1932. 